\title{
Boundary value problems for strongly nonlinear equations under a Wintner-Nagumo growth condition
}

\section{Cristina Marcelli* and Francesca Papalini}

\author{
"Correspondence: \\ marcelli@dipmat.univpm.it \\ Department of Mathematical \\ Sciences, Polytechnic University of \\ Marche, Via Brecce Bianche, \\ Ancona, 60131, Italy
}

\begin{abstract}
We study the following strongly nonlinear differential equation:

$$
\left(a(t, x(t)) \Phi\left(x^{\prime}(t)\right)\right)^{\prime}=f\left(t, x(t), x^{\prime}(t)\right), \quad \text { a.e. in }[0, T]
$$

subjected to various boundary conditions including, as particular cases, the classical Dirichlet, periodic, Neumann and Sturm-Liouville problems. We adopt the method of lower and upper solutions requiring a weak form of a Wintner-Nagumo growth condition.
\end{abstract}

MSC: Primary 34B15; secondary 34B24; 34C25

Keywords: $\Phi$-Laplacian; second order differential equations; nonlinear boundary conditions; Sturm-Liouville conditions; periodic problems; Neumann problems; lower and upper solutions; fixed point techniques; nonlinear differential operators; Wintner-Nagumo growth condition

\section{Introduction}

Boundary value problems involving $\phi$-Laplacian-type operators have been intensively investigated (see, e.g., [1-4]), even for singular or non-surjective operators (see [5-8]). More recently, a certain interest has been devoted to differential operators involving also a nonlinear function of the state variable as in the following equation:

$$
\left(a(x(t)) \Phi\left(x^{\prime}(t)\right)\right)^{\prime}=f\left(t, x(t), x^{\prime}(t)\right), \quad \text { a.e. in } I=[0, T],
$$

where $a$ is a continuous positive function, $\phi$ is a strictly increasing homeomorphism and $f$ is a Carathéodory function. In this framework, results on the solvability of boundary value problems, both in compact intervals and on the whole real line, were established (see [9, 10]). Finally, for results concerning differential inclusions or non-autonomous differential operators, see $[11,12]$ and [13].

The usual technique in this context is generally based on the method of lower and upper solutions, combined with some Nagumo-type growth condition, which is needed to ensure an a priori bound for the derivatives of the solutions in order to apply a suitable fixed point result. In the above quoted papers, the presence of the nonlinear term $a$ inside the

(c) The Author(s) 2017. This article is distributed under the terms of the Creative Commons Attribution 4.0 International License (http://creativecommons.org/licenses/by/4.0/), which permits unrestricted use, distribution, and reproduction in any medium, provided you give appropriate credit to the original author(s) and the source, provide a link to the Creative Commons license, and indicate if changes were made. 
differential operator influences the requirements about the differential operator $\Phi$, which was assumed to be homogeneous, or having at most linear growth at infinity.

In the recent paper [4], the authors obtain existence and multiplicity results for Dirichlet, periodic and Neumann problems for the equation

$$
\Phi\left(x^{\prime}(t)\right)^{\prime}=f\left(t, x(t), x^{\prime}(t)\right), \quad \text { a.e. in }[0, T]
$$

under the following Wintner-Nagumo condition:

$$
|f(t, x, y)| \leq \psi(|y|)\left(\ell(t)+c(t)|y|^{(p-1) / p}\right)
$$

where $\ell \in L^{1}(I), c \in L^{p}(I), \psi$ satisfying $\int^{\infty} \frac{1}{\psi\left(\left|\phi^{-1}(s)\right|\right)} \mathrm{d} s=+\infty$, which is weaker than other Nagumo-type conditions previously considered in other papers such as $[8,10,12]$.

Motivated by this, in the present paper we investigate the following general equation:

$$
\left(a(t, x(t)) \Phi\left(x^{\prime}(t)\right)\right)^{\prime}=f\left(t, x(t), x^{\prime}(t)\right), \quad \text { a.e. in }[0, T]
$$

involving a positive continuous function $a$, under growth condition (2). We show that this condition suffices to obtain existence results also for the general equation (3) and, mainly, it allows us to widen the class of operators $\Phi$. Indeed, we are now able to treat very general differential operators, not necessarily homogeneous, nor having polynomial growth, which are only required to be strictly increasing homeomorphisms.

In this context, by using a different approach with respect to [4], we are able to prove existence results for solutions of (1) subjected to very general boundary value conditions including, as particular cases, Dirichlet, periodic, Sturm-Liouville and Neumann problems. Our results extend those in [4] both for the presence of the function $a$ inside the differential operators and for the great generality of the structure on the boundary conditions. Finally, we also provide some examples of application of our results, in which the operator $\Phi$ is not homogeneous and grows exponentially at infinity.

\section{Preliminaries and auxiliary results}

Let us consider the differential equation

$$
\left(a(t, x(t)) \Phi\left(x^{\prime}(t)\right)\right)^{\prime}=f\left(t, x(t), x^{\prime}(t)\right) \quad \text { a.e. } t \in I=[0, T]
$$

where $a: I \times \mathbb{R} \rightarrow \mathbb{R}$ is a positive continuous function, $\Phi: \mathbb{R} \rightarrow \mathbb{R}$ is an increasing homeomorphism and $f: I \times J \times \mathbb{R} \rightarrow \mathbb{R}$, with $J \subset \mathbb{R}$ an interval, is a Carathéodory function, that is, $f(\cdot, x, y)$ is measurable for every $x, y \in J \times \mathbb{R}$ and $f(t, \cdot, \cdot)$ is continuous for a.e. $t \in I$.

In this context, a solution for equation (4) is a function $x \in C^{1}(I)$ with $x(t) \in J$ for every $t \in I$ such that the map $t \mapsto a(t, x(t)) \Phi\left(x^{\prime}(t)\right)$ is absolutely continuous in $I$ and $\left(a(t, x(t)) \Phi\left(x^{\prime}(t)\right)\right)^{\prime}=f\left(t, x(t), x^{\prime}(t)\right)$ for a.e. $t \in I$.

In what follows, we investigate the existence of solutions for equation (4) satisfying different boundary conditions. Our approach is based on fixed point techniques suitably combined to the method of upper and lower solutions, according to the following definition. 
A lower solution [upper solution] for equation (4) is a function $\alpha \in C^{1}(I)$ such that $\alpha(t) \in$ $J$, the map $t \mapsto a(t, \alpha(t)) \Phi\left(x^{\prime}(t)\right)$ is absolutely continuous in $I$ and

$$
\left(a(t, \alpha(t)) \Phi\left(\alpha^{\prime}(t)\right)\right)^{\prime} \geq[\leq] f\left(t, \alpha(t), \alpha^{\prime}(t)\right) \quad \text { a.e. } t \in I .
$$

A well-ordered pair of upper and lower solutions in I for equation (4) is a pair $\alpha, \beta \in C^{1}(I)$ where $\alpha$ is a lower solution, $\beta$ is an upper solution and $\alpha(t) \leq \beta(t)$ for every $t \in I$. For every pair of well-ordered lower and upper solutions, the functional interval $[\alpha, \beta]$ is defined as follows:

$$
[\alpha, \beta]=\left\{x \in C^{1}(I): \alpha(t) \leq x(t) \leq \beta(t), \forall t \in I\right\}
$$

Finally, we will adopt the following notations relatively to real numbers $x, y$ :

$$
x \wedge y:=\min \{x, y\}, \quad x \vee y:=\max \{x, y\}, \quad x^{+}:=x \vee 0, \quad x^{-}:=(-x)^{+} .
$$

The fixed point technique which will be used is based on an existence result for the following functional differential boundary value problem:

$$
\left\{\begin{array}{l}
\left(A_{u}(t) \Phi\left(u^{\prime}(t)\right)\right)^{\prime}=F_{u}(t) \quad \text { a.e. in } I=[0, T] \\
u(0)=v_{1}, \quad u(T)=v_{2},
\end{array}\right.
$$

where $v_{1}, v_{2} \in \mathbb{R}$ are given constants, $\Phi: \mathbb{R} \rightarrow \mathbb{R}$ is an increasing homeomorphism, and $A: C^{1}(I) \rightarrow C(I), x \mapsto A_{x}, F: C^{1}(I) \rightarrow L^{1}(I), x \mapsto F_{x}$, are continuous functionals. In [8, Theorem 1], the following existence result was proved.

Theorem 1 Assume that the functional $A$ is uniformly continuous and maps bounded sets into uniformly continuous sets of $C(I)$, that is, for every bounded set $B \subset C^{1}(I)$ and every $\varepsilon>0$, there exists a real number $\delta=\delta_{\varepsilon, B}$ such that

$$
\left|A_{x}\left(t_{1}\right)-A_{x}\left(t_{2}\right)\right|<\varepsilon \quad \text { for all } x \in B, t_{1}, t_{2} \in I \text { with }\left|t_{1}-t_{2}\right|<\delta \text {. }
$$

Moreover, assume that there exist $m, M>0$ such that

$$
m \leq A_{x}(t) \leq M \quad \text { for every } x \in C^{1}(I), t \in I .
$$

Finally, suppose that there exists $\phi \in L^{1}(I)$ such that

$$
\left|F_{x}(t)\right| \leq \phi(t) \quad \text { for every } x \in C^{1}(I) \text {, a.e. } t \in I \text {. }
$$

Then, for every $v_{1}, v_{2} \in \mathbb{R}$, there exists a function $u \in C^{1}(I)$ with $A_{u} \cdot\left(\Phi \circ u^{\prime}\right) \in W^{1,1}(I)$, a solution of problem (5).

Remark 1 In [8] the previous result was given in the context of research involving differential operators satisfying $\Phi(0)=0$; however, as it is easy to check, this assumption is not necessary in order to prove Theorem 1 . 


\section{The Dirichlet problem}

The study of the solvability of (4) under various boundary conditions starts with the classical Dirichlet problem, that is,

$$
\left\{\begin{array}{l}
\left(a(t, x(t)) \Phi\left(x^{\prime}(t)\right)\right)^{\prime}=f\left(t, x(t), x^{\prime}(t)\right) \quad \text { a.e. } t \in I, \\
x(0)=c, \quad x(T)=d
\end{array}\right.
$$

where $c, d \in \mathbb{R}$ are given. The following result states the existence of a solution for problem (D) belonging to the functional interval delimited by a well-ordered pair of lower and upper solutions.

Theorem 2 Assume that, for all $r>0$, there exists $\gamma_{r} \in L^{1}(I)$ such that

$$
|f(t, x, y)| \leq \gamma_{r}(t) \quad \text { for a.e. } t \in I \text {, every } x, y \text { with }|x|,|y| \leq r .
$$

Suppose that there exists a measurable function $\psi:(0,+\infty) \rightarrow(0,+\infty)$ such that

$$
\int^{+\infty} \frac{\mathrm{d} s}{\psi(s)}=+\infty
$$

and assume that there exist positive constants $H, p \in(1,+\infty]$ and positive functions $v \in$ $L^{p}(I), \ell \in L^{1}(I)$ such that

$$
|f(t, x, y)| \leq \psi(a(t, x)|\Phi(y)|) \cdot\left(\ell(t)+v(t)|y|^{\frac{p-1}{p}}\right),
$$

for a.e. $t \in I$, every $x \in J$ and $|y|>H$, with the convention $\frac{p-1}{p}=1$ if $p=+\infty$.

Finally, assume that there exists a well-ordered pair $\alpha$ and $\beta$ of lower and upper solutions in I for equation (4).

Then, for every $c, d \in \mathbb{R}$ such that $\alpha(0) \leq c \leq \beta(0), \alpha(T) \leq d \leq \beta(T)$, problem (D) has a solution $x_{c, d}$ belonging to the functional interval $[\alpha, \beta]$, that is,

$$
\alpha(t) \leq x_{c, d}(t) \leq \beta(t) \quad \text { for all } t \in I
$$

Moreover, for every $M>0$, there exists a constant $\Lambda=\Lambda(M, H, v, \ell, \psi)$ such that if $\|\alpha\|_{C(I)} \leq M,\|\beta\|_{C(I)} \leq M,\left\|\alpha^{\prime}\right\|_{C(I)} \leq \Lambda$ and $\left\|\beta^{\prime}\right\|_{C(I)} \leq \Lambda$, then for every $c \in[\alpha(0), \beta(0)], d \in$ $[\alpha(T), \beta(T)]$, we have

$$
\left\|x_{c, d}\right\|_{C(I)} \leq M \text { and }\left\|x_{c, d}^{\prime}\right\|_{C(I)} \leq \Lambda
$$

Proof Let $M>0$ be a constant such that $\|\alpha\|_{C(I)} \leq M,\|\beta\|_{C(I)} \leq M$ and put

$$
m_{0}:=\min _{(t, x) \in I \times[-M, M]} a(t, x), \quad m_{1}:=\max _{(t, x) \in I \times[-M, M]} a(t, x) .
$$

Since $\Phi$ is an increasing homeomorphism, it is possible to choose a constant $L>0$ such that

$$
L>\max \{H, 2 M / T\}, \quad \Phi(L)>0, \quad \Phi(-L)<0 .
$$


Moreover, by (10) there exists a constant $N>L$ such that

$$
\Phi(N)>\frac{m_{1}}{m_{0}} \Phi(L), \quad \Phi(-N)<\frac{m_{1}}{m_{0}} \Phi(-L),
$$

and

$$
\begin{aligned}
& \int_{m_{1} \Phi(L)}^{m_{0} \Phi(N)} \frac{\mathrm{d} s}{\psi(s)}>\|\ell\|_{1}+(2 M)^{1-\frac{1}{p}}\|v\|_{p}, \\
& \int_{-m_{1} \Phi(-L)}^{-m_{0} \Phi(-N)} \frac{\mathrm{d} s}{\psi(s)}>\|\ell\|_{1}+(2 M)^{1-\frac{1}{p}}\|v\|_{p} .
\end{aligned}
$$

Let us now introduce the truncation operator $T: W^{1,1}(I) \rightarrow W^{1,1}(I)$ defined by

$$
T(x):=T_{x} \quad \text { where } T_{x}(t):=[\beta(t) \wedge x(t)] \vee \alpha(t)
$$

$T$ is well defined and $T_{x}^{\prime}(t)=x^{\prime}(t)$ for a.a. $t \in I$ such that $\alpha(t)<x(t)<\beta(t)$, whereas $T_{x}^{\prime}(t)=$ $\alpha^{\prime}(t)$ for a.e. $t$ such that $x(t) \leq \alpha(t), T_{x}^{\prime}(t)=\beta^{\prime}(t)$ for a.e. $t$ such that $x(t) \geq \beta(t)$.

Let us consider the modified function $f^{*}: I \times \mathbb{R}^{2} \rightarrow \mathbb{R}$ defined by

$$
f^{*}(t, x, y)= \begin{cases}f\left(t, \beta(t), \beta^{\prime}(t)\right)+\arctan (x-\beta(t)) & \text { if } x>\beta(t) \\ f(t, x, y) & \text { if } \alpha(t) \leq x \leq \beta(t) \\ f\left(t, \alpha(t), \alpha^{\prime}(t)\right)+\arctan (x-\alpha(t)) & \text { if } x<\alpha(t)\end{cases}
$$

and the following auxiliary boundary value problem

$$
\left\{\begin{array}{l}
\left(a\left(t, T_{x}(t)\right) \Phi\left(x^{\prime}(t)\right)\right)^{\prime}=f^{*}\left(t, x(t), q_{N}\left(T_{x}^{\prime}(t)\right)\right) \quad \text { a.e. } t \in I, \\
x(0)=c, \quad x(T)=d
\end{array}\right.
$$

where $q_{N}(r):=(-N) \vee[r \wedge N]$ for all $r \in \mathbb{R}$.

Claim 1 There exists a solution to problem (19).

Let $A: C^{1}(I) \rightarrow C(I)$ be the functional defined as

$$
x \mapsto A_{x}, \quad A_{x}(t):=a\left(t, T_{x}(t)\right) .
$$

Of course, $A$ is well defined, bounded and, from the uniform continuity of $a$ in $I \times[-M, M]$, $A$ is also uniformly continuous. Moreover, if $B$ is a bounded subset of $C^{1}(I)$, i.e., there exists $k>0$ such that $\|x\|_{C^{1}(I)} \leq k$ for all $x \in B$. Then, fixed $\epsilon>0$, by the uniform continuity of $a(\cdot, \cdot)$ in $I \times[-M, M]$, there exists $\delta=\delta(\epsilon)>0$ such that

$$
\left|a\left(t_{1}, \xi_{1}\right)-a\left(t_{2}, \xi_{2}\right)\right|<\epsilon \quad \text { whenever }\left|t_{1}-t_{2}\right|<\delta,\left|\xi_{1}-\xi_{2}\right|<\delta
$$

Put $k^{*}:=\max \left\{\|\alpha\|_{C^{1}},\|\beta\|_{C^{1}}, k\right\}$ and $\eta:=\min \left\{\frac{\delta}{k^{*}}, \delta\right\}$, if $\left|t_{1}-t_{2}\right|<\eta$, we have

$$
\left|T_{x}\left(t_{1}\right)-T_{x}\left(t_{2}\right)\right| \leq\left|\int_{t_{1}}^{t_{2}}\right| T_{x}^{\prime}(s)|\mathrm{d} s| \leq k^{*}\left|t_{1}-t_{2}\right|<\delta \quad \text { for every } x \in B
$$


So, we deduce that $\left|A_{x}\left(t_{1}\right)-A_{x}\left(t_{2}\right)\right|<\epsilon$ for all $x \in B$ provided that $\left|t_{1}-t_{2}\right|<\eta$. So the functional $A$ satisfies assumption (6).

Now let $F: C^{1}(I) \rightarrow L^{1}(I), x \mapsto F_{x}$, be the functional defined by

$$
F_{x}=f^{*}\left(t, x(t), q_{N}\left(T_{x}^{\prime}(t)\right)\right), \quad \text { a.e. on } \mathrm{I} \text {. }
$$

Of course, $F$ is continuous. Moreover, put

$$
\phi(t):=\gamma_{M+N}(t)+\left|f\left(t, \alpha(t), \alpha^{\prime}(t)\right)\right|+\left|f\left(t, \beta(t), \beta^{\prime}(t)\right)\right|+\frac{\pi}{2},
$$

we have that $\phi \in L^{1}(I)$ by (9) and $\left|F_{x}(t)\right| \leq \phi(t)$ for almost all $t \in I$ and all $x \in C^{1}(I)$ by (9) and (18). Therefore, $F$ satisfies hypothesis (8). So, by applying Theorem 1 , there exists a function $u \in C^{1}(I)$ such that, for all $t \in I, A_{u} \cdot\left(\Phi \circ u^{\prime}\right) \in W^{1,1}(I)$ and

$$
\left\{\begin{array}{l}
\left(A_{u}(t) \Phi\left(u^{\prime}(t)\right)\right)^{\prime}=F_{u}(t) \quad \text { a.e. on } I \\
u(0)=c, \quad u(T)=d
\end{array}\right.
$$

Obviously, $u$ is a solution of problem (19).

Claim 2 The solution $u$ of problem (19) belongs to the functional interval $[\alpha, \beta]$.

We prove that $\alpha(t) \leq u(t)$ for every $t \in I$. In a similar way it can be proved that $u(t) \leq \beta(t)$ for all $t \in I$. Assume, by contradiction, that there exists $t_{0} \in I$ such that $u\left(t_{0}\right)-\alpha\left(t_{0}\right)=$ $\min _{t \in I}(u(t)-\alpha(t))<0$. Then, from the boundary conditions, $t_{0} \in(0, T)$ and there exists an interval $\left[t_{1}, t_{2}\right] \subset I$, containing $t_{0}$, such that $u\left(t_{1}\right)-\alpha\left(t_{1}\right)=u\left(t_{2}\right)-\alpha\left(t_{2}\right)=0$ and $u(t)-\alpha(t)<0$ for every $t \in\left(t_{1}, t_{2}\right)$. Hence, $u^{\prime}\left(t_{0}\right)-\alpha^{\prime}\left(t_{0}\right)=0, T_{u}(t) \equiv \alpha(t)$ for every $t \in\left(t_{1}, t_{2}\right)$. Then, from (18) and the definition of lower solution, for a.e. $t \in\left(t_{1}, t_{2}\right)$, we get

$$
\begin{aligned}
\left(a(t, \alpha(t)) \Phi\left(u^{\prime}(t)\right)\right)^{\prime} & =\left(a\left(t, T_{u}(t)\right) \Phi\left(u^{\prime}(t)\right)\right)^{\prime}=f^{*}\left(t, u(t), q_{N}\left(T_{u}^{\prime}(t)\right)\right) \\
& =f\left(t, \alpha(t), \alpha^{\prime}(t)\right)+\arctan (u(t)-\alpha(t)) \\
& <f\left(t, \alpha(t), \alpha^{\prime}(t)\right) \leq\left(a(t, \alpha(t)) \Phi\left(\alpha^{\prime}(t)\right)\right)^{\prime} .
\end{aligned}
$$

Thus, the function $t \mapsto a(t, \alpha(t))\left(\Phi\left(u^{\prime}(t)\right)-\Phi\left(\alpha^{\prime}(t)\right)\right)$ is strictly decreasing in $\left[t_{1}, t_{2}\right]$. Consequently,

$$
a(t, \alpha(t))\left(\Phi\left(u^{\prime}(t)\right)-\Phi\left(\alpha^{\prime}(t)\right)\right)<a\left(t_{0}, \alpha\left(t_{0}\right)\right)\left(\Phi\left(u^{\prime}\left(t_{0}\right)\right)-\Phi\left(\alpha^{\prime}\left(t_{0}\right)\right)\right)=0
$$

for $t \in\left[t_{0}, t_{2}\right]$; therefore also $\Phi\left(u^{\prime}(t)\right)-\Phi\left(\alpha^{\prime}(t)\right)<0$. Recalling that $\Phi$ is strictly monotone, we get $u^{\prime}(t)<\alpha^{\prime}(t)$ in $\left[t_{0}, t_{2}\right]$. Hence, we get

$$
u\left(t_{2}\right)-\alpha\left(t_{2}\right)<u\left(t_{0}\right)-\alpha\left(t_{0}\right)<0
$$

which contradicts the definition of the interval $\left[t_{1}, t_{2}\right]$. Then also Claim 2 is proved.

As a consequence of Claim 2, recalling the definitions of the modified function $f^{*}$ (see (18)) and of the truncation operator $T$ (see (17)), we derive that the solution $u$ satisfies

$$
\left(a(t, u(t)) \Phi\left(u^{\prime}(t)\right)^{\prime}=f\left(t, u(t), q_{N}\left(u^{\prime}(t)\right)\right) \quad \text { a.e. } t \in I .\right.
$$


Claim $3\left|u^{\prime}(t)\right| \leq N$ for every $t \in I$.

Notice that if $H<\left|u^{\prime}(t)\right| \leq N$, from (11), we obtain

$$
\left|\left(a(t, u(t)) \Phi\left(u^{\prime}(t)\right)\right)^{\prime}\right| \leq \psi\left(a(t, u(t))\left|\Phi\left(u^{\prime}(t)\right)\right|\right) \cdot\left(\ell(t)+v(t)\left|u^{\prime}(t)\right|^{\frac{p-1}{p}}\right) .
$$

Observe now that from (14) and the mean value theorem there exists $\hat{t} \in(0, T)$ such that

$$
\left|u^{\prime}(\hat{t})\right|=\left|\frac{d-c}{T}\right| \leq \frac{2 M}{T}<L<N .
$$

Assume, by contradiction, that $\max \left\{u^{\prime}(t): t \in I\right\} \geq N$ (in a similar way we can argue if $\left.\min \left\{u^{\prime}(t): t \in I\right\} \leq-N\right)$, then there exists an interval $\left(\tau_{0}, \tau_{1}\right) \subset I$ such that $L<u^{\prime}(t)<N$ in $\left(\tau_{0}, \tau_{1}\right)$ and $u^{\prime}\left(\tau_{0}\right)=L$ and $u^{\prime}\left(\tau_{1}\right)=N$ or vice versa. From (13), (15), (21) and using the Holder inequality, we obtain

$$
\begin{aligned}
\int_{m_{1} \Phi(L)}^{m_{0} \Phi(N)} \frac{\mathrm{d} s}{\psi(s)} & \leq \int_{a\left(u\left(\tau_{0}\right)\right) \Phi\left(u^{\prime}\left(\tau_{0}\right)\right)}^{a\left(u\left(\tau_{1}\right) \Phi\left(u^{\prime}\left(\tau_{1}\right)\right)\right.} \frac{\mathrm{d} s}{\psi(s)}=\int_{\tau_{0}}^{\tau_{1}} \frac{\left(a(t, u(t)) \Phi\left(u^{\prime}(t)\right)\right)^{\prime}}{\psi\left(a(t, u(t)) \Phi\left(u^{\prime}(t)\right)\right)} \mathrm{d} t \\
& \leq \int_{\tau_{0}}^{\tau_{1}} \frac{\left|\left(a(t, u(t)) \Phi\left(u^{\prime}(t)\right)\right)^{\prime}\right|}{\psi\left(a(t, u(t)) \Phi\left(u^{\prime}(t)\right)\right)} \mathrm{d} t \leq \int_{\tau_{0}}^{\tau_{1}}\left(\ell(t)+v(t)\left|u^{\prime}(t)\right|^{\frac{p-1}{p}}\right) \mathrm{d} t \\
& \leq\|\ell\|_{1}+\|v\|_{p}\left|u\left(\tau_{1}\right)-u\left(\tau_{0}\right)\right|^{\frac{p-1}{p}} \\
& \leq\|\ell\|_{1}+\|v\|_{p}(2 M)^{1-\frac{1}{p}}
\end{aligned}
$$

in contradiction with (16), and also Claim 3 is proved.

Summarizing, taking account of (20), we conclude that $x_{c, d}:=u$ is a solution of problem (D) satisfying (12).

As we mentioned in Introduction, the Wintner-Nagumo condition (11) is weaker than similar growth conditions assumed in many papers related to boundary value problems of similar type, also with respect to results in which the differential operator does not contain the function $a$. Moreover, it allows us to widen the range of the differential operators $\Phi$ we can consider that can be very general, not necessarily homogeneous, nor having polynomial growth. For instance, in the following example, we apply the existence result to an operator $\Phi$ having exponential growth.

Example 1 Let us consider the following equation:

$$
\left(a(t, x(t)) \sinh \left(x^{\prime}(t)\right)\right)^{\prime}=\left(\sinh \left(x^{\prime}(t)\right)+1\right)\left[h(t)(x(t)+b(t))+g(x(t)) x^{\prime}(t)\right]
$$

where $a \in C([0, T] \times \mathbb{R}), h \in L^{1}([0, T]), b \in C([0, T]), g \in C(\mathbb{R})$ are given generic functions, with $a(t, x)>0$ for every $(t, x) \in I \times \mathbb{R}$ and $h(t) \geq 0$ for every $t \in[0, T]$.

Observe that for a fixed $K$ such that $|b(t)| \leq K$ in $[0, T]$, the constant functions $\alpha(t):=-K$ and $\beta(t):=K$ are a well-ordered pair of lower and upper solutions. Moreover, put

$$
f(t, x, y):=(\sinh y+1)[h(t)(x+b(t))+g(x) y]
$$


it is immediate to verify that $f$ is a Carathéodory function such that, whenever $|x| \leq r$ and $|y| \leq r$, we have

$$
|f(t, x, y)| \leq(\sinh r+1)\left[h(t)(r+b(t))+r \max _{x \in[-r, r]}|g(x)|\right]:=\gamma_{r}(t)
$$

with $\gamma_{r} \in L^{1}([0, T])$, and assumption (9) is satisfied.

Furthermore, put $M:=\max _{x \in[-K, K]} g(x)$ and $m:=\min _{(t, x) \in I \times[-M, M]} a(t, x)$, since

$$
\begin{aligned}
|f(t, x, y)| & \leq(|\sinh y|+1)[2 K h(t)+M|y|] \\
& \leq\left(\frac{a(t, x)}{m}|\sinh y|+1\right)[2 K h(t)+M|y|]
\end{aligned}
$$

whenever $x \in[-K, K]$ and, for every $y \in \mathbb{R}$, we get that assumptions (10) and (11) are satisfied choosing $\psi(s):=\frac{s}{m}+1, \ell(t):=2 K h(t), v(t):=M$ and $p=+\infty$. So, for every $c, d \in$ $[-K, K]$, problem $(\mathrm{D})$ admits a solution.

The following example shows an equation governed by a differential operator of the type considered in [10] and [8], and with a right-hand side which satisfies condition (11), but not other Nagumo-type growth conditions as those assumed in the mentioned papers.

Example 2 Consider the following differential equation:

$$
\left(a(t, x(t)) x^{\prime}(t)\right)^{\prime}=\left(x^{\prime}(t)+1\right)\left[h(t)(x(t)+b(t))+g(x(t)) x^{\prime}(t)\right] .
$$

With the same notations as in Example 1, we get that assumptions (9), (10) and (11) are satisfied and Theorem 2 can be applied. On the contrary, in order to apply the results in [10] there should exist a function $\theta$ such that $|f(t, x, y)|=|y+1| \cdot|y| \leq \theta(|y|)$, with $\int^{+\infty} \frac{1}{\theta(s)} \mathrm{d} s=+\infty$, which is not possible.

\section{General nonlinear boundary conditions}

We consider now more general boundary conditions in order to deal with periodic, Neumann, Sturm-Liouville boundary conditions for equation (4), using the result obtained in the previous section for Dirichlet problems, following the idea developed in [1]. To this aim, in what follows we adopt the notation $\left(D_{c, d}\right)$ to denote the Dirichlet problem $(D)$, when we need to emphasize the values of the boundary conditions.

The following lemma provides a compactness-type result for the solutions of equation (4) obtained by means of Theorem 2 .

Lemma 1 Let assumption (9) be satisfied, and let $\alpha, \beta$ be a well-ordered pair of lower and upper solutions for equation (4). Then, for every pair of sequences $\left(c_{n}\right)_{n}$ and $\left(d_{n}\right)_{n}$ of real numbers satisfying $c_{n} \in[\alpha(0), \beta(0)]$ and $d_{n} \in[\alpha(T), \beta(T)]$ for every $n \in \mathbb{N}$, and for every sequence $\left(x_{n}\right)_{n}$ of solutions of problem $D_{c_{n}, d_{n}}$, equibounded in $C^{1}$ and belonging to the functional interval $[\alpha, \beta]$, there exists a subsequence $\left(x_{n_{k}}\right)_{k}$ such that

$$
x_{n_{k}}(t) \rightarrow x_{0}(t), \quad x_{n_{k}}^{\prime}(t) \rightarrow x_{0}^{\prime}(t) \quad \text { uniformly in } I
$$


Proof Let $\left(c_{n}\right)_{n},\left(d_{n}\right)_{n}$ be a pair of sequences of real numbers satisfying $\alpha(0) \leq c_{n} \leq \beta(0)$ and $\alpha(T) \leq d_{n} \leq \beta(T)$ for every $n \in \mathbb{N}$, and let $\left(x_{n}\right)_{n}$ be a sequence of solutions of problem $D_{c_{n}, d_{n}}$, equibounded in $C^{1}$ and belonging to the functional interval $[\alpha, \beta]$. We can assume without restriction, possibly by passing to subsequences, that $c_{n} \rightarrow c_{0}, d_{n} \rightarrow d_{0}$. By the equiboundedness of the sequence $\left(x_{n}\right)_{n}$ we have $\left|x_{n}(t)\right|+\left|x_{n}^{\prime}(t)\right|<r$ for all $t \in I$, for some $r>0$. Therefore, by assumption (9) we get

$$
\left|\left(a\left(t, x_{n}(t)\right) \Phi\left(x_{n}^{\prime}(t)\right)\right)^{\prime}\right|=\left|f\left(t, x_{n}(t), x_{n}^{\prime}(t)\right)\right| \leq \gamma_{r}(t) \quad \text { for all } n \text {, a.e. } t \in I \text {. }
$$

So, the sequence of functions $\left(a\left(t, x_{n}(t)\right) \Phi\left(x_{n}^{\prime}(t)\right)\right)_{n}$ is equicontinuous on $I$. Moreover, since the function $1 / a(t, x)$ is uniformly continuous on $I \times[-r, r]$, we deduce that also the sequence $\left(1 / a\left(t, x_{n}(t)\right)\right)_{n}$ is equicontinuous on $I$. Therefore, the sequence $\left(\Phi\left(x_{n}^{\prime}(t)\right)\right)_{n}$ is equicontinuous on $I$, as it is the product of equicontinuous functions. Finally, by the continuity of the function $\Phi^{-1}$, we deduce that the sequence $\left(x_{n}^{\prime}\right)_{n}$ is uniformly continuous on $I$. Recalling that $\left|x_{n}^{\prime}(t)\right| \leq r$ for every $n \in \mathbb{N}$ and a.e. $t \in I$, by the AscoliArzelà theorem, we infer the existence of a subsequence $\left(x_{n_{k}}^{\prime}\right)_{k}$ uniformly convergent to a continuous function $y_{0}$, implying that also $\left(x_{n_{k}}\right)_{k}$ uniformly converges to the function $x_{0}(t)=\int_{0}^{t} y_{0}(s) \mathrm{d} s$.

Moreover, notice that, for all $t \in I$, we have

$$
a\left(t, x_{n_{k}}(t)\right) \Phi\left(x_{n_{k}}^{\prime}(t)\right)=a\left(0, x_{n_{k}}(0)\right) \Phi\left(x_{n_{k}}^{\prime}(0)\right)+\int_{0}^{t} f\left(s, x_{n_{k}}(s), x_{n_{k}}^{\prime}(s)\right) \mathrm{d} s .
$$

Hence, by the dominated convergence theorem we obtain

$$
a\left(t, x_{0}(t)\right) \Phi\left(x_{0}^{\prime}(t)\right)=a\left(0, x_{0}(0)\right) \Phi\left(x_{0}^{\prime}(0)\right)+\int_{0}^{t} f\left(s, x_{0}(s), x_{0}^{\prime}(s)\right) \mathrm{d} s
$$

that is, $x_{0}$ is a solution of (4).

In order to handle various types of boundary condition, let us consider the following problem with very general nonlinear boundary conditions:

$$
\left\{\begin{array}{l}
\left(a(t, x(t)) \Phi\left(x^{\prime}(t)\right)\right)^{\prime}=f\left(t, x(t), x^{\prime}(t)\right) \quad \text { a.e. } t \in I \\
g\left(x(0), x(T), x^{\prime}(0), x^{\prime}(T)\right)=0 \\
x(T)=h(x(0))
\end{array}\right.
$$

where $g: \mathbb{R}^{4} \rightarrow \mathbb{R}$ and $h: \mathbb{R} \rightarrow \mathbb{R}$ are continuous functions.

By applying Theorem 2 we are able to prove an existence result also for the general problem (22).

Theorem 3 Suppose that there exists a well-ordered pair $\alpha, \beta$ of lower and upper solutions for equation (4) such that

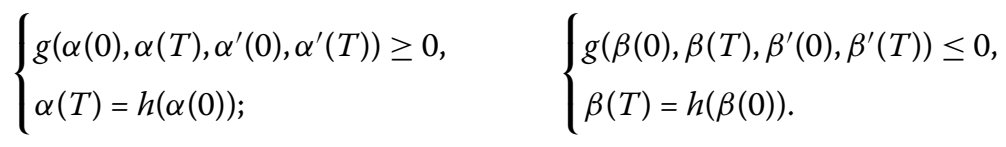


Let assumptions (9), (10), (11) be satisfied. Moreover, suppose $h$ is increasing and

$$
g(u, v, \cdot, z) \quad \text { is increasing; } \quad g(u, v, w, \cdot) \quad \text { is decreasing. }
$$

Then problem (22) admits a solution $x$, belonging to the functional interval $[\alpha, \beta]$, such that $\|x\|_{C^{1}} \leq \Lambda$, where $\Lambda$ is the constant given by Theorem 2 (see (12)), with respect to $M:=\max \left\{\max _{t \in I}|\alpha(t)|, \max _{t \in I}|\beta(t)|\right\}$.

Proof Let us fix $c \in[\alpha(0), \beta(0)]$. By the monotonicity of $h$, we have $\alpha(T) \leq h(c) \leq \beta(T)$; so, by Theorem 2 , the problem $\left(D_{c, h(c)}\right)$ admits a solution $x_{c}$ belonging to the functional interval $[\alpha, \beta]$. Moreover, there exists a constant $\Lambda$ such that, for every $c \in[\alpha(0), \beta(0)]$, we have (see (12))

$$
\left\|x_{c}\right\|_{C(I)} \leq M \quad \text { and } \quad\left\|x_{c}^{\prime}\right\|_{C(I)} \leq \Lambda
$$

Put

$$
\begin{aligned}
\Omega:= & \left\{c \in[\alpha(0), \beta(0)]: \text { there exists a solution } x_{c} \in[\alpha, \beta] \text { of }\left(D_{c, h(c)}\right)\right. \\
& \text { satisfying (24) and such that } \left.g\left(x_{c}(0), x_{c}(T), x_{c}^{\prime}(0), x_{c}^{\prime}(T)\right) \geq 0\right\} .
\end{aligned}
$$

Notice that $\Omega$ is not empty because $\alpha(0) \in \Omega$. Indeed, if $c=\alpha(0)$, then also $x_{c}(T)=\alpha(T)$ and, since $\alpha(t) \leq x_{c}(t)$, for all $t \in I$, we get $x_{c}^{\prime}(0) \geq \alpha^{\prime}(0)$ and $x_{c}^{\prime}(T) \leq \alpha^{\prime}(T)$. Thus, from (23), we have

$$
g\left(x_{c}(0), x_{c}(T), x_{c}^{\prime}(0), x_{c}^{\prime}(T)\right) \geq 0 \text {. }
$$

Let $c^{*}:=\sup \Omega$, and let us prove that $c^{*} \in \Omega$. This is trivial if $c^{*}=\alpha(0)$, whereas, if $c^{*}>\alpha(0)$, let $\left(c_{n}\right)_{n} \subset \Omega$ be a sequence converging to $c_{*}$ from below. Put $d_{n}=h\left(c_{n}\right)$, by Theorem 2 , for every $n \in \mathbb{N}$, there exists a solution $x_{n}$ of problem $\left(D_{c_{n}, d_{n}}\right)$, belonging to the functional interval $[\alpha, \beta]$, such that $x_{n}$ satisfies (24) for every $n \in \mathbb{N}$. So, we can apply Lemma 1 to infer the existence of a subsequence $\left(x_{n_{k}}\right)_{k}$ of solutions with the properties

$$
x_{n_{k}}(t) \rightarrow x_{*}(t), \quad x_{n_{k}}^{\prime}(t) \rightarrow x_{*}^{\prime}(t) \quad \text { for every } t \in I
$$

for some solution $x_{*}$ of equation (4). Of course, $c^{*}:=x_{*}(0)$ and, by the continuity of $h$, we get that $x_{*}$ is a solution of problem $\left(D_{c_{*}, h\left(c_{*}\right)}\right)$. Moreover, since $c_{n_{k}} \in \Omega$, we have $g\left(x_{n_{k}}(0), x_{n_{k}}(T), x_{n_{k}}^{\prime}(0), x_{n_{k}}^{\prime}(T)\right) \geq 0$ for every $k \in \mathbb{N}$ and, by the continuity of the function $g$, we obtain

$$
g\left(x_{*}(0), x_{*}(T), x_{*}^{\prime}(0), x_{*}^{\prime}(T)\right) \geq 0,
$$

then $c_{*} \in \Omega$ and so $c_{*}=\max \Omega$.

If $c^{*}=\beta(0)$, then $x_{*}^{\prime}(0) \leq \beta^{\prime}(0)$. Moreover, $x_{*}(T)=h(\beta(0))=\beta(T)$ implying $x_{*}^{\prime}(T) \geq$ $\beta^{\prime}(T)$. Hence, by (23) we deduce

$$
\begin{aligned}
g\left(x_{*}(0), x_{*}(T), x_{*}^{\prime}(0), x_{*}^{\prime}(T)\right) & =g\left(\beta(0), \beta(T), x_{*}^{\prime}(0), x_{*}^{\prime}(T)\right) \\
& \leq g\left(\beta(0), \beta(T), \beta^{\prime}(0), \beta^{\prime}(T)\right) \leq 0 .
\end{aligned}
$$


So, taking account of (25), we get $g\left(x_{*}(0), x_{*}(T), x_{*}^{\prime}(0), x_{*}^{\prime}(T)\right)=0$ and $x_{*}$ is a solution of problem (22).

Let us now treat the case $c^{*}<\beta(0)$. In order to do this, let us consider a decreasing sequence $\left(c_{m}\right)_{m}$ converging to $c^{*}$. Of course, the functions $x_{*}$ and $\beta$ can be considered as a well-ordered pair of lower and upper solutions for equation (4), with $\left\|x^{*}\right\|_{C(I)} \leq M$ and $\left\|x^{*^{\prime}}\right\|_{C(I)} \leq \Lambda$. By applying again Theorem 2 , we deduce that, for every $m \in \mathbb{N}$, there exists a solution $\xi_{m}$ of problem $\left(D_{c_{m}, h\left(c_{m}\right)}\right)$ belonging to the functional interval $\left[x_{*}, \beta\right]$, again with $\left\|\xi_{m}\right\|_{C(I)} \leq M$ and $\left\|\xi_{m}^{\prime}\right\|_{C(I)} \leq \Lambda$ for every $m \in \mathbb{N}$. Hence, we can apply Lemma 1 again, obtaining the existence of a subsequence $\left(\xi_{m_{k}}\right)_{k}$ of solutions such that

$$
\xi_{m_{k}}(t) \rightarrow \xi_{*}(t), \quad \xi_{m_{k}}^{\prime}(t) \rightarrow \xi_{*}^{\prime}(t) \quad \text { for every } t \in I
$$

for some solution $\xi_{*}$ of equation (4). By the continuity of $h$, the function $\xi_{*}$ is a solution of problem $\left(D_{c_{*}, h\left(c_{*}\right)}\right)$. Moreover, since $c_{m_{k}}>c_{*}=\max \Omega$, we have $c_{m} \notin \Omega$ for every $m \in$ $\mathbb{N}$. Since $\left\|\xi_{m}\right\|_{C^{1}(I)} \leq \Lambda$, necessarily we have $g\left(\xi_{m}(0), \xi_{m}(T), \xi_{m}^{\prime}(0), \xi_{m}^{\prime}(T)\right)<0$. Thus, by the continuity of $g$, we have

$$
g\left(\xi_{*}(0), \xi_{*}(T), \xi_{*}^{\prime}(0), \xi_{*}^{\prime}(T)\right) \leq 0 .
$$

Nevertheless, being $\xi_{*}(t) \geq x_{*}(t)$ for every $t \in I$, with $\xi_{*}(0)=x_{*}(0)$ and $\xi_{*}(t)=x_{*}(T)$, we deduce that $\xi_{*}^{\prime}(0) \geq x_{*}^{\prime}(0)$ and $\xi_{*}(T) \leq x_{*}^{\prime}(T)$. So, by (23) and (25) we infer

$$
\begin{aligned}
g\left(\xi_{*}(0), \xi_{*}(T), \xi_{*}^{\prime}(0), \xi_{*}^{\prime}(T)\right) & =g\left(x_{*}(0), x_{*}(T), \xi_{*}^{\prime}(0), \xi_{*}^{\prime}(T)\right) \\
& \geq g\left(x_{*}(0), x_{*}(T), x_{*}^{\prime}(0), x_{*}^{\prime}(T)\right) \geq 0
\end{aligned}
$$

that jointly with (26) implies

$$
g\left(\xi_{*}(0), \xi_{*}(T), \xi_{*}^{\prime}(0), \xi_{*}^{\prime}(T)\right)=0
$$

and $\xi_{*}$ is a solution of problem (22).

The general boundary conditions considered in problem (22) include, as a particular case, periodic boundary conditions, that is, the problem

$$
\left\{\begin{array}{l}
\left(a(t, x(t)) \Phi\left(x^{\prime}(t)\right)\right)^{\prime}=f\left(t, x(t), x^{\prime}(t)\right) \quad \text { a.e. } t \in I, \\
x(0)=x(T), \quad x^{\prime}(0)=x^{\prime}(T) .
\end{array}\right.
$$

As an immediate consequence of Theorem 3, the following existence result holds.

Theorem 4 Let $\alpha$ and $\beta$ be a well-ordered pair of lower and upper solutions for equation (4) such that

$$
\left\{\begin{array} { l } 
{ \alpha ( 0 ) = \alpha ( T ) , } \\
{ \alpha ^ { \prime } ( 0 ) \geq \alpha ^ { \prime } ( T ) , }
\end{array} \text { and } \quad \left\{\begin{array}{l}
\beta(0)=\beta(T), \\
\beta^{\prime}(0) \leq \beta^{\prime}(T) .
\end{array}\right.\right.
$$

Assume that hypotheses (9), (10), (11) are satisfied. Then problem (27) has a solution belonging to the functional interval $[\alpha, \beta]$. 
Proof The assertion is an immediate consequence of Theorem 3, taking $g(u, v, w, z):=w-z$ and $h(r):=r$.

Let us consider now the following boundary value problem:

$$
\left\{\begin{array}{l}
\left(a(t, x(t)) \Phi\left(x^{\prime}(t)\right)\right)^{\prime}=f\left(t, x(t), x^{\prime}(t)\right) \quad \text { a.e. } t \in I, \\
p\left(x(0), x^{\prime}(0)\right)=0, \quad q\left(x(T), x^{\prime}(T)\right)=0,
\end{array}\right.
$$

where $p, q: \mathbb{R}^{2} \rightarrow \mathbb{R}$ are continuous functions. The following existence result for problem (28) holds.

Theorem 5 Let $\alpha$ and $\beta$ be a well-ordered pair of lower and upper solutions for equation (4) such that

$$
\left\{\begin{array} { l } 
{ p ( \alpha ( 0 ) , \alpha ^ { \prime } ( 0 ) ) \geq 0 , } \\
{ q ( \alpha ( T ) , \alpha ^ { \prime } ( T ) ) \geq 0 ; }
\end{array} \quad \text { and } \quad \left\{\begin{array}{l}
p\left(\beta(0), \beta^{\prime}(0)\right) \leq 0 \\
q\left(\beta(T), \beta^{\prime}(T)\right) \leq 0
\end{array}\right.\right.
$$

Assume that hypotheses (9), (10), (11) are satisfied. Moreover, assume that, for every $s \in \mathbb{R}$, we have

$$
p(s, \cdot) \quad \text { is increasing and } q(s, \cdot) \text { is decreasing. }
$$

\section{Then problem (28) has a solution belonging to the functional interval $[\alpha, \beta]$.}

Proof For every $c \in[\alpha(0), \beta(0)]$ and $d \in[\alpha(T), \beta(T)]$, let $x_{c, d}$ denote a solution of problem $\left(D_{c, d}\right)$ belonging to the functional interval $[\alpha, \beta]$, whose existence is ensured by Theorem 2 satisfying (12).

For every fixed $d \in[\alpha(T), \beta(T)]$, let us consider now the following auxiliary boundary value problem:

$$
\left\{\begin{array}{l}
\left(a(t, x(t)) \Phi\left(x^{\prime}(t)\right)\right)^{\prime}=f\left(t, x(t), x^{\prime}(t)\right) \quad \text { a.e. } t \in I, \\
x(T)=d, \quad p\left(x(0), x^{\prime}(0)\right)=0 .
\end{array}\right.
$$

So, by virtue of Theorem 3, we have that, for every $d \in[\alpha(T), \beta(T)]$, there exists a solution $y_{d}$ of problem $\left(Q_{d}\right)$, belonging to the functional interval $[\alpha, \beta]$, such that $\|y\|_{C(I)} \leq M$ and $\left\|y^{\prime}\right\|_{C(I)} \leq \Lambda$.

Observe now that, taking account of (29), when $d=\alpha(T)$, we have $q\left(y(T), y^{\prime}(T)\right) \geq$ $q\left(\alpha(T), \alpha^{\prime}(T)\right) \geq 0$ for every solution $y$ of problem $\left(Q_{d}\right)$; whereas for $d=\beta(T)$, we have $q\left(y(T), y^{\prime}(T)\right) \leq q\left(\beta(T), \beta^{\prime}(T)\right) \leq 0$ for every solution $y$ of problem $\left(Q_{d}\right)$. Our goal is to show that there exists a value $d^{*} \in[\alpha(T), \beta(T)]$ and a solution $y_{*}$ of problem $\left(Q_{d^{*}}\right)$ such that $q\left(y_{d^{*}}(T), y_{d^{*}}^{\prime}(T)\right)=0$.

To this aim, put

$$
\begin{aligned}
\Upsilon:= & \left\{d \in[\alpha(T), \beta(T)]: \text { there exists a solution } \xi_{d} \text { of problem }\left(Q_{d}\right)\right. \\
& \text { such that } \left.\left\|\xi_{d}\right\|_{C(I)} \leq M,\left\|\xi_{d}^{\prime}\right\|_{C(I)} \leq \Lambda \text { and } q\left(\xi_{d}(T), \xi_{d}^{\prime}(T)\right) \geq 0\right\} .
\end{aligned}
$$


We have that $\alpha(T) \in \Upsilon$, so $\Upsilon \neq \emptyset$. Let $d^{*}:=\sup \Upsilon$, and let us prove that $d^{*}=\max \Upsilon$, that is, $q\left(\xi_{*}(T), \xi_{*}^{\prime}(T)\right) \geq 0$ for some solution $\xi_{*}$ of problem $\left(Q_{d^{*}}\right)$. This is trivial if $d^{*}=$ $\alpha(T)$, whereas if $d^{*}>\alpha(T)$, let $\left(d_{n}\right)_{n} \subset \Upsilon$ be an increasing sequence converging to $d^{*}$, and let $\left(\xi_{d_{n}}\right)_{n}$ be a sequence of solutions of problem $\left(Q_{d_{n}}\right)$ satisfying $q\left(\xi_{d_{n}}(T), \xi_{d_{n}}^{\prime}(T)\right) \geq 0$. By virtue of Lemma 1 we get the existence of a subsequence $\left(\xi_{d_{n_{k}}}\right)_{k}$ such that

$$
\xi_{d_{n_{k}}}(t) \rightarrow \xi_{*}(t), \quad \xi_{d_{n_{k}}}^{\prime}(t) \rightarrow \xi_{*}^{\prime}(t) \quad \text { for every } t \in I
$$

for some solution $\xi_{*}$ of equation (4). By the continuity of $p$ and $q$, we get $\xi_{*}$ is a solution of problem $\left(Q_{d^{*}}\right)$ such that $q\left(\xi_{*}(T), \xi_{*}^{\prime}(T)\right) \geq 0$, hence $d^{*} \in \Upsilon$.

If $d_{*}=\beta(T)$, then $\xi_{*}^{\prime}(T) \geq \beta^{\prime}(T)$ and by (29) we get

$$
q\left(\xi_{*}(t), \xi_{*}^{\prime}(T)\right)=q\left(\beta(T), \xi_{*}^{\prime}(T)\right) \leq q\left(\beta(T), \beta^{\prime}(T)\right) \leq 0
$$

So, $\xi_{*}$ is a solution of problem $(Q)$.

Finally, let us treat the case $d^{*}<\beta(T)$. Let $\left(d_{m}\right)_{m}$ be a decreasing sequence converging to $d^{*}$. Notice that the functions $\xi_{*}$ and $\beta$ are a well-ordered pair of lower and upper solutions for equation (4) satisfying the assumptions of Theorem 3 (for $h(c):=d$ and $g(u, v, w, z):=$ $p(u, w))$. So, for every $m \in \mathbb{N}$, there exists a solution $\zeta_{m}$ of problem $G$ such that

$$
\xi_{*}(t) \leq \zeta_{m}(t) \leq \beta(t) \quad \text { for every } t \in I
$$

Since $d_{m} \notin \Upsilon$, we have

$$
q\left(\zeta_{m}(T), \zeta_{m}^{\prime}(T)\right)<0 \quad \text { for every } m \in \mathbb{N} \text {. }
$$

We can apply again Lemma 1 to deduce the existence of a subsequence $\left(\zeta_{m_{k}}\right)_{k}$ such that

$$
\zeta_{m_{k}} \rightarrow \zeta_{*}(t), \quad \zeta_{m_{k}}^{\prime}(t) \rightarrow \zeta_{*}^{\prime}(t) \quad \text { for every } t \in I
$$

and

$$
\xi_{*}(t) \leq \zeta_{*}(t) \leq \beta(t) \quad \text { for every } t \in I,
$$

for some $\zeta_{*}$ solution of equation (4). By the continuity of the function $p$, we have $p\left(\zeta_{*}(0), \zeta_{*}^{\prime}(0)\right)=0$, and by (30) and the continuity of $q$, we get

$$
q\left(\zeta_{*}(0), \zeta_{*}^{\prime}(0)\right) \leq 0
$$

On the other hand, since $\xi_{*}(T)=d^{*}=\zeta_{*}(T)$, by $(31)$ we get $\zeta_{*}^{\prime}(T) \leq \xi_{*}^{\prime}(T)$. Therefore, from (29) we infer

$$
q\left(\zeta_{*}(T), \zeta_{*}^{\prime}(T)=q\left(\xi_{*}(T), \zeta_{*}^{\prime}(T)\right) \geq q\left(\xi_{*}(T), \xi_{*}^{\prime}(T)\right) \geq 0\right.
$$

that jointly with (32) implies $q\left(\zeta_{*}(0), \zeta_{*}^{\prime}(0)\right)=0$. So, $\zeta_{*}$ is a solution of problem $(Q)$. 
Remark 2 The boundary conditions in problem (28) cover, as particular cases, both the Sturm-Liouville and the Neumann problems. In fact, for the former problem, one takes $p(s, t):=\ell_{0} s+m_{0} t-v_{0}$ and $q(s, t)=\ell_{1} s-m_{1} t-v_{1}$, with $m_{0}, m_{1} \geq 0$. With this choice, one gets the boundary conditions

$$
\ell_{0} x(0)+m_{0} x^{\prime}(0)=v_{0}, \quad \ell_{1} x(T)-m_{1} x^{\prime}(T)=v_{1} .
$$

For the latter problem, one takes $p(s, t):=t-v_{0}$ and $q(s, t):=v_{1}-t$, and problem (28) becomes the Neumann problem

$$
\left\{\begin{array}{l}
\left(a(t, x(t)) \Phi\left(x^{\prime}(t)\right)\right)^{\prime}=f\left(t, x(t), x^{\prime}(t)\right) \quad \text { a.e. } t \in I, \\
x^{\prime}(0)=v_{0}, \quad x^{\prime}(T)=v_{1} .
\end{array}\right.
$$

Thus, Theorem 5 gives a condition for the existence of solutions for both these problems.

\section{Conclusions}

We have proved an existence result for the following strongly nonlinear differential equation:

$$
\left(a(t, x(t)) \Phi\left(x^{\prime}(t)\right)\right)^{\prime}=f\left(t, x(t), x^{\prime}(t)\right), \quad \text { a.e. in }[0, T]
$$

subjected to very general boundary conditions (see Theorems 3 and 5), covering, as particular cases, the classical Dirichlet, Neumann, periodic and Sturm-Liouville problems. Here the function $a=a(t, x)$ is generic (it is only required to be continuous and positive) and mainly the differential operator $\Phi$ is quite general, not necessarily homogeneous, nor having polynomial growth. Finally, we also present some examples of applications of these results, for which the results in the previous literature in the matter are not applicable.

Funding

Not applicable.

\section{Abbreviations}

Not applicable

Availability of data and materials

Not applicable.

Ethics approval and consent to participate

Not applicable.

Competing interests

The authors declare that they have no competing interests.

Authors' contributions

All authors read and approved the final manuscript. The contribution of the authors is equal.

\section{Publisher's Note}

Springer Nature remains neutral with regard to jurisdictional claims in published maps and institutional affiliations. 
References

1. Cabada, A, Pouso, RL: Existence results for the problem $\left(\phi\left(u^{\prime}\right)\right)^{\prime}=f\left(t, u, u^{\prime}\right)$ with periodic and Neumann boundary conditions. Nonlinear Anal. TMA 30, 1733-1742 (1997)

2. Cabada, A, Pouso, RL: Existence results for the problem $\left(\phi\left(u^{\prime}\right)\right)^{\prime}=f\left(t, u, u^{\prime}\right)$ with nonlinear boundary conditions. Nonlinear Anal. TMA 35, 221-231 (1999)

3. Cabada, A, O'Regan, D, Pouso, RL: Second order problems with functional conditions including Sturm-Liouville and multipoint conditions. Math. Nachr. 281, 1254-1263 (2008)

4. El Khattabi, N, Frigon, M, Ayyadi, N: Multiple solutions of boundary value problems with $\phi$-Laplacian operators and under a Wintner-Nagumo growth condition. Bound. Value Probl. 2013, 236 (2013)

5. Bereanu, C, Mawhin, J: Existence and multiplicity results for some nonlinear problems with singular $\Phi$-Laplacian. J. Differ. Equ. 243, 536-557 (2007)

6. Bereanu, C, Mawhin, J: Periodic solutions of nonlinear perturbations of $\Phi$-Laplacians with possibly bounded $\Phi$ Nonlinear Anal. TMA 68, 1668-1681 (2008)

7. Bereanu, C, Mawhin, J: Boundary value problems for some nonlinear systems with singular $\Phi$-Laplacian. J. Fixed Point Theory Appl. 4(1), 57-75 (2008)

8. Ferracuti, L, Papalini, F: Boundary value problems for strongly nonlinear multivalued equations involving different $\Phi$-Laplacians. Adv. Differ. Equ. 14, (5-6) (2009)

9. Calamai, A: Heteroclinic solutions of boundary value problems on the real line involving singular $\Phi$-Laplacian operators. J. Math. Anal. Appl. 378(2), 667-679 (2011)

10. Cupini, G, Marcelli, C, Papalini, F: Heteroclinic solutions of boundary-value problems on the real line involving general nonlinear differential operators. Differ. Integral Equ. 24(7-8), 619-644 (2011)

11. Ferracuti, L, Marcelli, C, Papalini, F: Boundary value problems for highly nonlinear inclusions governed by non-surjective $\Phi$-Laplacians. Set-Valued Var. Anal. 19(1), 1-21 (2011)

12. Marcelli, C: The role of boundary data on the solvability of some equations involving non-autonomous, nonlinear differential operators. Bound. Value Probl. 2013, 252 (2013)

13. Minhós, F: Sufficient conditions for the existence of heteroclinic solutions for $\phi$-Laplacian differential equations. Complex Var. Elliptic Equ. 62, 123-134 (2017)

\section{Submit your manuscript to a SpringerOpen ${ }^{\circ}$ journal and benefit from:}

- Convenient online submission

- Rigorous peer review

- Open access: articles freely available online

- High visibility within the field

- Retaining the copyright to your article

Submit your next manuscript at $\boldsymbol{\triangleright}$ springeropen.com 